\title{
Front Matter: Volume 11430
}

, "Front Matter: Volume 11430," Proc. SPIE 11430, MIPPR 2019: Pattern Recognition and Computer Vision, 1143001 (14 February 2020); doi: $10.1117 / 12.2565844$

SDIE Event: Eleventh International Symposium on Multispectral Image Processing and Pattern Recognition (MIPPR2019), 2019, Wuhan, China 


\title{
MIPPR 2019: Pattern Recognition and Computer Vision
}

\author{
Nong Sang \\ Jayaram K. Udupa \\ Yuehuan Wang \\ Zhenbing liu \\ Editors
}

2-3 November 2019

Wuhan, China

Organized by

Huazhong University of Science and Technology (China)

National Key Laboratory of Science and Technology on Multi-spectral Information Processing (China)

Wuhan Institute of Technology (China)

Sponsored by

National Key Laboratory of Science and Technology on Multi-spectral Information Processing (China)

Huazhong University of Science and Technology (China)

Wuhan Institute of Technology (China)

Automation Association of Hubei (China)

Published by

SPIE

Volume 11430 
The papers in this volume were part of the technical conference cited on the cover and title page. Papers were selected and subject to review by the editors and conference program committee. Some conference presentations may not be available for publication. Additional papers and presentation recordings may be available online in the SPIE Digital Library at SPIEDigitalLibrary.org.

The papers reflect the work and thoughts of the authors and are published herein as submitted. The publisher is not responsible for the validity of the information or for any outcomes resulting from reliance thereon.

Please use the following format to cite material from these proceedings:

Author(s), "Title of Paper," in MIPPR 2019: Pattern Recognition and Computer Vision, edited by Nong Sang, Jayaram K. Udupa, Yuehuan Wang, Zhenbing Liu, Proceedings of SPIE Vol. 11430 (SPIE, Bellingham, WA, 2020) Seven-digit Article CID Number.

ISSN: 0277-786X

ISSN: 1996-756X (electronic)

ISBN: 9781510636378

ISBN: 9781510636385 (electronic)

Published by

SPIE

P.O. Box 10, Bellingham, Washington 98227-0010 USA

Telephone +1 3606763290 (Pacific Time) · Fax +1 3606471445

SPIE.org

Copyright (c) 2020, Society of Photo-Optical Instrumentation Engineers.

Copying of material in this book for internal or personal use, or for the internal or personal use of specific clients, beyond the fair use provisions granted by the U.S. Copyright Law is authorized by SPIE subject to payment of copying fees. The Transactional Reporting Service base fee for this volume is $\$ 21.00$ per article (or portion thereof), which should be paid directly to the Copyright Clearance Center (CCC), 222 Rosewood Drive, Danvers, MA 01923 . Payment may also be made electronically through CCC Online at copyright.com. Other copying for republication, resale, advertising or promotion, or any form of systematic or multiple reproduction of any material in this book is prohibited except with permission in writing from the publisher. The CCC fee code is 0277$786 \mathrm{X} / 20 / \$ 21.00$.

Printed in the United States of America by Curran Associates, Inc., under license from SPIE.

Publication of record for individual papers is online in the SPIE Digital Library.

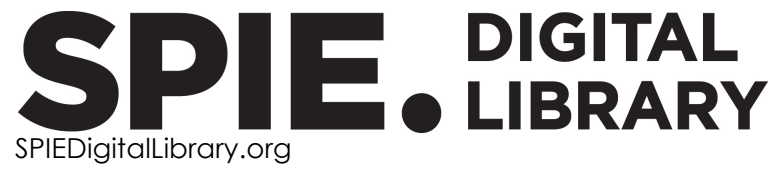

Paper Numbering: Proceedings of SPIE follow an e-First publication model. A unique citation identifier (CID) number is assigned to each article at the time of publication. Utilization of CIDs allows articles to be fully citable as soon as they are published online, and connects the same identifier to all online and print versions of the publication. SPIE uses a seven-digit CID article numbering system structured as follows:

- The first five digits correspond to the SPIE volume number.

- The last two digits indicate publication order within the volume using a Base 36 numbering system employing both numerals and letters. These two-number sets start with 00, 01, 02, 03, 04 , $05,06,07,08,09,0 A, 0 B \ldots$ OZ, followed by 10-1Z, 20-2Z, etc. The CID Number appears on each page of the manuscript. 


\title{
Contents
}

\author{
vii Authors \\ ix Symposium Committee \\ xiii Introduction
}

PATTERN RECOGNITION AND COMPUTER VISION

$1143002 \quad$ Heterogeneous features extraction based on deep learning for drug-related webpages classification [1 1430-1]

1143003 Research on vehicle detection method for multi-polarimetric SAR image based on scene analysis [11430-2]

$1143005 \quad$ Blind deblurring of Gaussian blurred images by blurred edge image [1 1430-6]

1143006 Performance evaluation of SIFT under low light contrast [1 1430-7]

1143007 Research on binocular real-time ranging method in window area [11430-8]

1143008 Cloud bottom height estimation methods for optical imaging terminal guidance [11430-9]

$114300 \mathrm{~A} \quad$ Rural settlements extraction based on deep learning from high spatial resolution remote sensing imagery [11430-12]

11430 OC G-CNN type recognition of typical aircraft based on target characteristics [11430-19]

11430 OD Semantic segmentation network combined with edge detection for building extraction in remote sensing images [11430-21]

$11430 \mathrm{OE} \quad$ Multi-scales feature integration single shot multi-box detector on small object detection [11430-22]

11430 OF Space objects attitude discrimination via light-curve measurements and deep convolutional neural networks [1 1430-23]

11430 OG Pose estimation for non-cooperative targets using 3D feature correspondences grouped via local and global constraints [11430-24]

$11430 \mathrm{OH} \quad$ Bidirectional LSTM-CRF models for keyword extraction in Chinese sport news [11430-25]

1143001 A method of adaptive learning rate tracking for embedded device based correlation surface evaluation [11430-26] 
$11430 \mathrm{OJ} \quad$ Object detection for rotated and densely arranged objects in aerial images using path aggregated feature pyramid networks [11430-27]

11430 OK Chinese news text classification based on attention-based CNN-BiLSTM [1 1430-28]

$11430 \mathrm{OL} \quad$ Delaunay growth algorithm based on point cloud curvature smoothing improvement [1 1430-29]

11430 OM Image categorization based on visual saliency and Bag-of-Words model [1 1430-32]

$114300 \mathrm{~N} \quad$ Research on improved meanshift algorithm based on fuzzy control principle [1 1430-33]

11430 OP Coupled discriminant mappings for heterogeneous face recognition [1 1430-37]

$114300 Q \quad$ ISAR target recognition system design based on artificial intelligence [1 1430-41]

11430 OR Hybrid feature fusion for person recognition in photo albums [1 1430-42]

11430 OT HOG and SVM algorithm based on vehicle model recognition [11430-44]

11430 OU Optimization calibration of fish-eye camera and its research in 3D reconstruction [11430-45]

11430 OV Dehazing network based on haze density [11430-46]

$11430 \mathrm{OW} \quad$ An improved patch matching algorithm based on fireworks algorithm [11430-48]

11430 OX Improved modality-invariant feature learning for degraded face recognition [11430-50]

11430 OY Detection and identification of insulator defects using hierarchical networks in the high-speed railway overhead contact system [11430-51]

$114300 Z$ An anomaly detection algorithm based on K-means and BP neural network in wireless sensor networks [11430-52]

1143013 Remote sensing image ship detection based on feature pyramid [1 1430-57]

1143014 Anti-interference recognition using 3D convolutional network with improved attention block [11430-60]

1143015 Infrared imaging simulation of atmospheric turbulence based on a improved GAN network method [11430-61]

1143016 An improved dynamic double threshold Canny edge detection algorithm [11430-62]

1143017 Semantic segmentation of buildings in high resolution remote sensing images using conditional random fields [1 1430-64] 
$114301 \mathrm{~B} \quad$ Real-time semantics based plane object tracking for augmented reality [1 1430-68]

$114301 \mathrm{C} \quad$ Image caption generation method based on adaptive attention mechanism [1 1430-69]

11430 1D A cascaded method for transmission tower number recognition in large scenes [11430-71]

$114301 \mathrm{E} \quad$ Turnover and shape filter based feature matching for image stitching [11430-73]

11430 1F Machine vision based 2D measurement method for industrial glass [11430-74]

$1143016 \quad$ Groundwater level abnormal detection based on correlation analysis [11430-75]

$114301 \mathrm{H} \quad$ Research and application of object recognition method based on depth neural network [11430-76]

$1143011 \quad$ Oracle-bone-inscription image segmentation based on simple fully convolutional networks [1 1430-77]

$114301 \mathrm{~K}$ A cross mosaic based partial fingerprint recognition strategy with multiple templates [11430-79]

$114301 \mathrm{~L} \quad$ Facial micro-expression recognition based on local region of the key frame [11430-80]

$114301 \mathrm{M} \quad$ Research on speech accurate recognition technology based on deep learning DNN-HMM [11430-81]

$114301 \mathrm{~N} \quad$ View prediction using manifold learning in non-linear feature subspace [1 1430-82]

1143010 ATSGPN: adaptive threshold instance segmentation network in 3D point cloud [11430-85]

$114301 Q \quad$ Increment adaptive correlation filter for visual tracking [1 1430-90]

$114301 R \quad$ Facial morphe via domain translation and FM²RLS [11430-91]

11430 is TLCS-Anchor: a new anchor strategy for detecting small-scale unmanned aerial vehicle [1 1430-92]

$114301 \mathrm{~T} \quad$ Image fusion for multimodality image via domain transfer and nonrigid transformation [11430-93]

$114301 \mathrm{~A} \quad$ A new aircraft classification algorithm based on sum pooling feature with remote sensing image [1 1430-94]

$114301 \mathrm{~V} \quad$ Crop extraction based on ultra-simple neural network modeling in the normalized RGB and CIE $\mathbf{L}^{*} \mathbf{a}^{*} \mathbf{b} *$ color spaces [1 1430-95]

$114301 \mathrm{~W} \quad$ Single image super-resolution based on enhanced deep residual GAN [1 1430-96] 
$114301 \mathrm{X} \quad$ Method of quality assessment based on convolution feature similarity for laser disturbing image [11430-97]

$114301 Y$ Line segment detection via random line fitting [1 1430-98]

1143012 Co-occurrence relationship encoding via channel merging for vehicle part recognition [11430-100]

1143020 Large margin multi-kernel tensor correlation filter for visual tracking [1 1430-102]

$1143021 \quad$ A new ground plane detection method based on continuous multi-frame images and hierarchical screening [11430-105]

1143022 A novel neural network approach for error transfer analysis in electronic instrument transformer [11430-106]

1143023 Large-scale book page retrieval by deep hashing networks [11430-107]

1143024 Object direction estimation by Constrained Convolutional Neural Network [11430-108]

1143025 Inference and sampling of gamma poisson process stick breaking construction model [11430-111] 


\section{Authors}

Numbers in the index correspond to the last two digits of the seven-digit citation identifier (CID) article numbering system used in Proceedings of SPIE. The first five digits reflect the volume number. Base 36 numbering is employed for the last two digits and indicates the order of articles within the volume. Numbers start with 00, 01, 02, 03, 04, 05, 06, 07, 08, 09, 0A, OB...0Z, followed by 10-12, 20-2Z, etc.

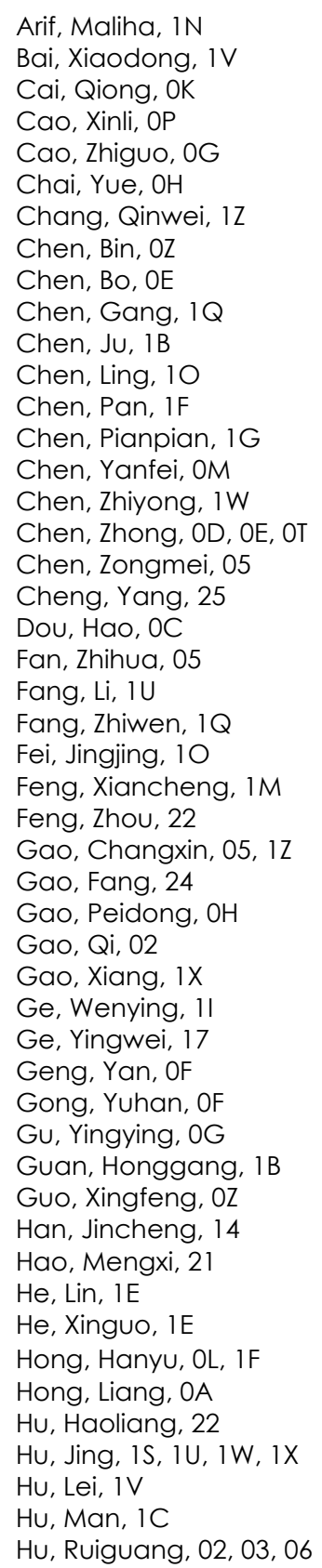

$\mathrm{Hu}, \mathrm{Zican}, \mathrm{OZ}$

Hua, Yanling, OV

Huang, Junchang, 22

Huang, Likun, $O P, O R$

Huang, Ruiqi, $\mathrm{OL}$

Huang, Ruojing, 1B

Huo, Jiao, 23

Ji, Kaixiang, OD

Jia, Yujiao, 02

Jiang, Kan, 1S

Jiang, Yiqi, $\mathrm{OH}$

Jiang, Zhongze, OD

Jin, Huazhong, $1 C$

Jin, Songzhi, 06, 21

Kang, SUSU, $1 \mathrm{U}$

Kang, Yanbing, $1 \mathrm{~K}$

Lei, Tao, $1 Y$

Li, Bingning, $O Q$

Li, Binzhe, $1 \mathrm{U}$

Li, Changfeng, 13

Li, Dehua, 25

Li, Jun, 07, OK

Li, Junqiang, OQ

Li, Lerenhan, 05

Li, Qi, OA

Li, Qingqing, 1C

Li, Qiong, $1 \mathrm{H}$

Li, Sheng, OR

Li, Wenhao, 05

$\mathrm{Li}$, Wenxiang, OM

Li, XiangJun, 1S, 1U, 1W, 1X

Li, Xiaofei, 22

$\mathrm{Li}$, Xinde, $0 \mathrm{~J}$

Li, Yacheng, 05

Li, Yanan, 1Q

Li, Zhimin, Ol

Liang, Shuai, 08

Liao, Jing, $1 \mathrm{~K}$

Liu, Bo, $1 Q$

Liu, Bo, $1 \mathrm{~V}$

Liu, Guoying, 11

Liu, Hong, OF

Liu, Jin, 24

Liv, Leyuan, 23

Liv, Meng, 08

Liu, Xiangyu, OJ

Liu, Yang, $O Q$

Liu, Yanxin, 02, 21

Lu, Xinxin, 1S 
Luo, Chuxuan, 24

Lv, Jing, 11

Ma, Xiaofeng, $1 \mathrm{H}$

Mahalanobis, Abhijit, $1 \mathrm{~N}$

Mao, Jiaxing, OC

Nie, Peng, 03

Nie, Shiwei, 13

Ou, Shichao, 1B

Pan, Hong, OJ

Pan, Rui, 22

Pei, Wei, OQ

Peng, Hongsheng, OM

Peng, Shaowu, 1B

Peng, Xiang, 22

Qi, Nie, 22

Qiu, Wu, 1M

Qu, Xin, 15

Ren, LiJun, $1 \mathrm{X}$

Sang, Nong, 05, 1 Z

Shang, Ke, IY

Shao, Xuehui, 03

Shi, Huili, OW

Shi, Ling, $1 \mathrm{~L}$

Song, Shuang, $1 \mathrm{E}$

Song, $X \cup, 11$

Song, Zhengxin, $\mathrm{OX}$

Sun, Huiling, OA

Sun, $Y \cup, 10$

Tang, Bing, OP, OR

Tian, Bin, $1 G$

Tian, Jinwen, $0 C, 1 Y$

Wan, Fang, $1 \mathrm{C}$

Wang, Guoyou, ID

Wang, Haihui, 07

Wang, Hao, 06

Wang, Hao, 20

Wang, Li, OG

Wang, Liya, OK

Wang, Meng, 07, OK

Wang, Quan, IY

Wang, Ran, ID

Wang, Xiaoke, OK

Wang, Yuehuan, 15

Wang, Zheng, 0N, 16

Wang, Zhicheng, 10

Wang, Zhongyang, 08

Wang, Ziwei, 07

Wei, Gang, 10

Wei, Xiaodan, 06

Wen, Ke, OP, OX

Wu, Kaizhi, $1 \mathrm{~K}$

Wu, Yifan, 15

Wu, Yu, 1C

Wu, Zechang, OM

Xia, Hang, $1 \mathrm{G}$

Xia, Wanyu, 1M

Xia, Yuanchun, 1D

Xiang, Houfan, $\mathrm{OZ}$

Xiao, Yang, $1 Q$

Xiao, Zhikang, ON, 16
Xie, Zhihua, $1 \mathrm{~L}$

Xiong, Tao, $1 \mathrm{~S}$

Xu, Guoxia, 20

Yan, LU, OU

Yan, Luxin, OW

Yang, Aiping, $1 \mathrm{~V}$

Yang, Bo, OY

Yang, Jian, OD, OE, OT

Yang, Jiaqi, OG

Yang, Jinkun, OT

Yang, Weidong, 13

Yang, Yicheng, $1 T$

Yang, Zhenqian, OF

Yang, Zhifang, OX

Yu, Cong, 20

Yu, Xinhe, $1 \mathrm{~L}$

Yu, Yu-Feng, 20

Yuan, Jun, $\mathrm{OZ}$

Yue, Mingyang, OY

Yue, Zhou, 1Q

Zhang, Bochuan, 03

Zhang, Changheng, OT

Zhang, Chonghua, IV

Zhang, Chunsen, $0 \mathrm{U}, 17$

Zhang, Cong, 06

Zhang, Hao, IY

Zhang, Hui, 21

Zhang, Jiahao, OE, OT

Zhang, Kun, IV

Zhang, Liubin, 07

Zhang, Siyu, ol

Zhang, Teng, $\mathrm{Ol}$

Zhang, Tianxu, 08

Zhang, Wei, OP, OR, IR

Zhang, Xian, $O Q$

Zhang, Xiuhua, IF

Zhang, Xuyang, IW

Zhang, YU, $1 Y$

Zhao, Congyu, OF

Zhao, Shuhan, IF

Zhao, Tongzhou, $\mathrm{OH}$

Zhao, Yi, 23

Zheng, WeiPing, $1 X$

Zhong, Sheng, Ol, OW, OY

Zhong, Weijun, OF

Zhou, Bin, 21

Zhou, Chen, IF

Zhou, Feng, ID

Zhou, Hongyu, 11

Zhou, Huabing, IR, IT

Zhou, Jianbang, OE

Zhou, Qianqian, OT

Zhou, Shifen, $1 G$

Zhou, Shiyang, 13

Zhu, Angfan, OG

Zong, Wenjun, $1 \mathrm{~L}$

Zou, Lamei, 13

Zou, Yang, 0N, 16

Zuo, Zhengrong, OV, 14 


\title{
Symposium Committees
}

\author{
Symposium Chairs \\ Deren Li, Wuhan University (China) \\ Bir Bhanu, University of California, Riverside (United States) \\ Program Committee Chairs \\ Jayaram K. Udupa, University of Pennsylvania (United States) \\ Tianxu Zhang, Huazhong University of Science and Technology \\ (China)
}

Program Committee

Christian Bauckhage, IAIS Fraunhofer (Germany)

Bir Bhanu, University of California, Riverside (United States)

Zhiguo Cao, Huazhong University of Science and Technology (China)

Chunqi Chang, Shenzhen University (China)

C. H. Chen, University of Massachusetts (United States)

Shaobo Chen, South Central University for Nationalities (China)

Xinjian Chen, Soochow University (China)

Melba M. Crawford, Purdue University (United States)

Armin B. Cremers, Universität Bonn (Germany)

He Deng, Wuhan Institute of Physics and Mathematics (China)

Mingyue Ding, Huazhong University of Science and Technology (China)

Aaron Fenster, The University of Western Ontario (Canada)

Wei Guo, Hebei Normal University (China)

Bruce E. Hirsch, Drexel University (United States)

Hanyu Hong, Wuhan Institute of Technology (China)

Xia Hua, Wuhan Institute of Technology (China)

Horace H.S. Ip, City University of Hong Kong (Hong Kong, China)

Jun Jo, Griffith University (Australia)

Irwin King, Chinese University of Hong Kong (Hong Kong, China)

Vladimir G. Krasilenko, Vinnitsa Social Economy Institute (Ukraine)

Xuelong Li, University of London (United Kingdom)

Qiang Li, University of Chicago (United States)

Senhu Li, Xoran Technologies LLC (United States)

Stan Z. Li, Chinese Academy of Sciences (China)

Xingde Li, Johns Hopkins University (United States)

Zicheng Li, Wuhan Institute of Technology (China)

Guoying Liu, Anyang Normal University (China) 
Jianguo Liu, Huazhong University of Science and Technology (China)

Xia Liu, Jianghan University (China)

Zhenbing Liu, Guilin University of Electronic Technology (China)

Hanqing Lu, Institute of Automation (China)

Henri Maître, École Nationale Supérieure des Télécommunications

(France)

Jiangqun Ni, Sun Yat-sen University (China)

Laszlo Nyul, University of Szeged (Hungary)

Chao Pan, Hubei University of Economics (China)

Shaohua Qu, Hubei University of Arts and Science (China)

Jonathan Roberts, Autonomous Systems Laboratory CSIRO ICT Centre (Australia)

Punam K. Saha, University of lowa (United States)

Nong Sang, Huazhong University of Science and Technology (China)

Xubang Shen, Chinese Academy of Sciences (China)

Yu Shi, Wuhan Institute of Technology (China)

M.V. Srinivasan, University of Queensland (Australia)

Hong Sun, Wuhan University (China)

Katarina Svanberg, Lund University (Sweden)

Jianjun Tan, Hubei University for Nationalities (China)

Dacheng Tao, Nanyang Technological University (Singapore)

Jay K. Udupa, University of Pennsylvania (United States)

Jinxue Wang, SPIE

Zhonghua Wang, Nanchang University of Aeronautics (China)

Baoming Wu, Third Military Medical University (China)

Hongan Wu, Chinese Academy of Surveying and Mapping (China)

Weichao Xu, Guangdong University of Technology (China)

Pingkun Yan, Philips Research North America (United States)

Hua Yang, Wuhan Polytechnic University (China)

Yuan Yuan, Aston University (United Kingdom)

Liangpei Zhang, Wuhan University (China)

Jun Zhang, Waseda University (Japan)

Tianxu Zhang, Huazhong University of Science and Technology

(China)

Sheng Zheng, China Three Gorges University (China)

Sheng Zhong, Huazhong University of Science and Technology (China)

Yanfei Zhong, Wuhan University (China)

Jie Zhou, Tsinghua University (China) 
Session Chairs

1 Multispectral Image Acquisition, Processing, and Analysis

Xinyu Zhang, Huazhong University of Science and Technology (China)

Chao Pan, Hubei University of Economics (China)

2 Automatic Target Recognition and Navigation

Bir Bhanu, University of California, Riverside (United States)

Guoying Liu, Anyang Normal University (China)

3 Pattern Recognition and Computer Vision

Jay K. Udupa, University of Pennsylvania (United States)

Jianguo Liu, Huazhong University of Science and Technology (China)

4 Parallel Processing of Images and Optimization Techniques; and Medical Imaging

Bruce E. Hirsch, Drexel University (United States)

He Deng, Wuhan Institute of Physics and Mathematics (China)

5 Remote Sensing, Image Processing, Geographic Information Systems, and Other Applications

Hanyu Hong, Wuhan Institute of Technology (China)

Nong Sang, Huazhong University of Science and Technology (China)

Organizing Committee Chair

Jianguo Liu, Huazhong University of Science and Technology (China)

Organizing Committee

Nong Sang, Huazhong University of Science and Technology (China)

Hongyan Wang, Huazhong University of Science and Technology

(China)

Luxin Yan, Huazhong University of Science and Technology (China)

General Secretary

Faxiong Zhang, Huazhong University of Science and Technology (China)

Associated General Secretaries

Xiaofeng Yue, Huazhong University of Science and Technology (China)

Jie Chen, Huazhong University of Science and Technology (China) 
Secretaries

Yuanchun Xia, Huazhong University of Science and Technology

(China)

Jun Xiong, Huazhong University of Science and Technology (China)

Feng Zhou, Huazhong University of Science and Technology (China)

Yi Zheng, Huazhong University of Science and Technology (China)

Hengrong Zhang, Huazhong University of Science and Technology (China)

Shuhong Xu, Huazhong University of Science and Technology (China)

Jinya Yu, Huazhong University of Science and Technology (China)

Jiaxin Xiong, Huazhong University of Science and Technology (China) 


\section{Introduction}

Welcome to the Proceedings of the 11 th International Symposium on Multispectral Image Processing and Pattern Recognition (MIPPR 2019) held 2-3 November 2019 in Wuhan, China.

MIPPR is a flagship biennial symposium which focuses mainly on the latest research in multispectral image processing and pattern recognition. The symposium has a broad charter. Multispectral is interpreted not just multiple-wavelength in a narrow sense but also multi-sensor, multi-modal, and multimedia. It covers many disciplines such as sensing, image processing, computer vision, pattern recognition and involves the development of efficient processing algorithms and their optimization and implementation. The wide range of applications considered in this symposium include automatic target recognition, autonomous navigation, medical image processing, remote sensing, geographic information systems and many others.

The symposium provides a forum for scientists, professors, engineers, and graduate students from universities, industries, and government laboratories to meet and exchange ideas and discuss theories, techniques, algorithms, and applications in multispectral image processing and pattern recognition. As expected, there were ample discussions both inside and outside the lecture halls helping to make MIPPR 2019 an exciting meeting.

In response to the Call for Papers, we received 258 submissions. Based on the reviews provided by an excellent program committee we accepted 199 papers covering many aspects of multispectral image processing and pattern recognition. To ensure a high-quality conference, all abstracts and Proceedings of SPIE papers are reviewed by the peers for technical merit and English expression. The proceedings from MIPPR 2019 consist of five volumes which will be included on the SPIE Digital Library.

- MIPPR 2019: Multispectral Image Acquisition, Processing, and Analysis (SPIE Volume 11428)

- MIPPR 2019: Automatic Target Recognition and Navigation (SPIE Volume 11429)

- MIPPR 2019: Pattern Recognition and Computer Vision (SPIE Volume 11430)

- MIPPR 2019: Parallel Processing of Images and Optimization Techniques; and Medical Imaging (SPIE Volume 11431)

- MIPPR 2019: Remote Sensing Image Processing, Geographic Information Systems, and Other Applications (SPIE Volume 11432).

The realization of a conference depends upon the hard work of many dedicated people. We would like to thank all the members of the Organizing Committee to 
put together this symposium for the benefit of all the researchers. They are responsible for making this conference a success. We hope the papers and the research results presented at this conference will inspire new research in all the areas related with multispectral image processing and pattern recognition.

Bir Bhanu 